\title{
COMPARISON OF TWO TYPES OF ORDER CONVERGENCE WITH TOPOLOGICAL CONVERGENCE IN AN ORDERED TOPOLOGICAL VECTOR SPACE
}

\author{
ROGER W. MAY ${ }^{1}$ AND CHARLES W. MCARTHUR
}

\begin{abstract}
Birkhoff and Peressini proved that if $(X, \mathcal{T})$ is a complete metrizable topological vector lattice, a sequence converges for the topology $\sigma$ iff the sequence relatively uniformly star converges. The above assumption of lattice structure is unnecessary. A necessary and sufficient condition for the conclusion is that the positive cone be closed, normal, and generating. If, moreover, the space $(X, \mathcal{T})$ is locally convex, Namioka [11, Theorem 5.4] has shown that $\mathcal{T}$ coincides with the order bound topology $\mathcal{T}_{b}$ and Gordon [4, Corollary, p. 423] (assuming lattice structure and local convexity) shows that metric convergence coincides with relative uniform star convergence. Omitting the assumptions of lattice structure and local convexity of $(X, \mathcal{T})$ it is shown for the nonnecessarily local convex topology $\sigma_{\mathrm{ru}}$ that $\sigma_{\mathrm{b}} \subset \mathcal{T}_{\mathrm{ru}}=\mathcal{T}$ and $\mathscr{T}_{\mathrm{b}}=\mathcal{T}_{\mathrm{ru}}=\sigma_{\text {when }}(X, \mathcal{T})$ is locally convex.
\end{abstract}

1. Introduction. The first of the two main results of this paper is a sharpening of a theorem of Birkhoff [1, Theorem 20, p. 370] and Peressini [12, Proposition 2.4, p. 162] which says that if $(X, \mathcal{T})$ is a complete metrizable topological vector lattice, then a sequence $\left(x_{n}\right)$ in $X$ converges to $x_{0}$ in $X$ for $\sigma$ if and only if $\left(x_{n}\right)$ relatively uniformly ${ }^{*}$-converges to $x_{0}$. The cone of positive elements of a topological vector lattice is closed, normal, and generating. We drop the irrelevant assumption of lattice structure and show that in a complete metrizable vector space ordered by a closed cone convergence in the metric topology is equivalent to relative uniform star convergence if and only if the cone is normal and generating. Klee [8], [11, Theorem 5.3] has shown that a closed cone in a complete metric space is generating if and only if it gives an open decomposition. We use this to prove that a closed cone in a complete metric space is generating iff metric convergence implies relative uniform star convergence of sequences. The second main result, Theorem 1.4, gives

Presented to the Society, June 18, 1976 under the title Comparison of order topologies with the topology of an ordered topological vector space; received by the editors, April 27, 1976 and, in revised form, August 16, 1976.

AMS (MOS) subject classifications (1970). Primary 46A40; Secondary 46A15.

Key words and phrases. Relative uniform convergence, order convergence, normal cone, generating cone, relatively uniform topology, order topology.

1 This work forms part of the first author's Doctoral Dissertation at Florida State University written under the direction of C. W. McArthur.

(1) American Mathematical Society 1977 
necessary and sufficient conditions for order star convergence to be equivalent to metric convergence.

The convergence theorems are then interpreted in terms of comparisons of the metric topology $\sigma$ with the order bound topology $\mathcal{T}_{\mathrm{b}}$, the relative uniform topology $\sigma_{\mathrm{ru}}$, and the order topology $\sigma_{\mathrm{o}}$. Theorems 1.2, 3.4, and 3.5, and Corollary 3.7 of this paper provide improvements or supplements to results of Gordon [4, Corollary, p. 423], Namioka [11, Theorem 5.4], and Carter and Ceitlin [2], [3], respectively.

First we generalize the notion of relative uniform convergence so as to apply to ordered vector spaces which are not lattices.

Definition 1.1. A sequence $\left(x_{n}\right)$ in an ordered vector space $(X, K)$ (where $K$ is the cone of positive elements) is relatively uniformly convergent to $x$ $\left(x_{n} \rightarrow^{(\mathrm{ru})} x\right)$ iff there exists a decreasing sequence $\left(\lambda_{n}\right)$ of real numbers with inf $\lambda_{n}=0$ and an element $z \in K$ such that $-\lambda_{n} z \leqslant x_{n}-x \leqslant \lambda_{n} z$ for $n=1,2, \ldots,\left(x_{n}\right)$ relatively uniformly ${ }^{*}$-converges to $x_{0}$ iff each subsequence of $\left(x_{n}\right)$ contains a subsequence which is relatively uniformly convergent to $x$ $\left(x_{n} \rightarrow^{\left(\mathrm{ru}^{*}\right)} x\right)$.

THEOREM 1.2. Let $(X, \mathcal{T})$ be a complete, metrizable topological vector space ordered by a $T$-closed cone $K$. The following statements are equivalent:

(1) $K$ is generating and normal.

(2) For each sequence $\left(x_{n}\right) \subset X$ and $x \in X, x_{n} \rightarrow^{\mathcal{T}} x$ iff $x_{n} \rightarrow^{\left(\mathrm{ru}^{*}\right)} x$.

A notion of convergence of sequences closely related to that of relative uniform convergence is order convergence.

Definition 1.3. Let $(X, K)$ be an ordered vector space. A sequence $\left(x_{n}\right) \subset X$ order converges to $x \in X\left(x_{n} \rightarrow^{\circ} x\right.$ or $x_{n}(0)$-converges to $\left.x\right)$ iff there exists a decreasing sequence $y_{n} \subset K$ such that inf $y_{n}=0\left(y_{n} \downarrow 0\right)$ and $-y_{n}$ $\leqslant x_{n}-x \leqslant y_{n}, n=1,2, \ldots ;$ a sequence $\left(x_{n}\right) \subset K$ order star converges to $x \in X\left(x_{n} \rightarrow^{\left(\mathrm{o}^{*}\right)} x\right.$ or $x_{n}\left(\mathrm{o}^{*}\right)$-converges to $\left.x\right)$ iff each subsequence of $\left(x_{n}\right)$ contains a subsequence which order converges to $x$.

It can be shown that our definition of order convergence agrees with that of Vulikh [13, p. 27], i.e., $x_{n} \rightarrow^{0} x$ iff there exist sequences $\left(u_{n}\right)$ and $\left(v_{n}\right)$ such that $u_{n}$ is decreasing with $x=\inf u_{n}, v_{n}$ is increasing with $x=\sup v_{n}$, and $v_{n} \leqslant x_{n}$ $\leqslant u_{n}, n=1,2, \ldots$

Clearly, if the ordering of a vector space is Archimedean, relative uniform convergence of a sequence implies order convergence of a sequence. Our second main result, Theorem 1.4 below, parallels the first.

THEOREM 1.4. Let $(X, \mathcal{T})$ be a complete, metrizable topological vector space ordered by a cone $K$. The following statements are equivalent:

(1) $K$ is $\mathcal{T}$-closed, generating, $\mathcal{T}$-normal and $(X, \mathcal{T})$ has property (A) (i.e., if $z_{n}$ is decreasing and inf $z_{n}=0$ then $z_{n} \rightarrow^{\top} \theta$ ).

(2) For each sequence $\left(x_{n}\right) \subset X$ and $x \in X, x_{n} \rightarrow^{\mathcal{T}} x$ iff $x_{n} \rightarrow^{\left(\mathrm{o}^{*}\right)} x$. 
The Banach space $l^{2}$, with its usual topology, ordered by the Lorentzian cone [12, p. 12]

$$
K=\left\{x=\left(x_{n}\right) \in l^{2} \mid 0 \leqslant x_{1} \text { and } \sum_{n=1}^{\infty} x_{n}^{2} \leqslant x_{1}^{2}\right\}
$$

provides an example of an ordered topological vector space which is not a lattice and yet whose cone $K$ is closed, normal, and generating. The space $\left(l^{2}, K\right)$ even has property (A) [10] so Theorems 1.2 and 1.4 apply to spaces which are not lattices.

\section{Proof of the main theorems.}

LEMMA 2.1. Let $(X, \mathcal{T})$ be a topological vector space ordered by a $\mathfrak{T}$-normal cone. Then for each sequence $\left(x_{n}\right) \subset X$ and $x \in X, x_{n} \rightarrow^{\left(\mathrm{ru}^{*}\right)} x$ implies $x_{n} \rightarrow^{\top} x$. If $(X, \mathcal{T})$ has property $(\mathrm{A})$ and the cone is $\mathcal{T}$-normal, then for each sequence $\left(x_{n}\right) \subset X$ and $x \in X, x_{n} \rightarrow^{\left(0^{*}\right)} x$ implies $x_{n} \rightarrow^{\text {T }} x$.

Proof. It will suffice to show that if $x_{n} \rightarrow^{\left(\mathrm{ru}^{*}\right)} \theta$, then $x_{n} \rightarrow^{\mathcal{T}} \theta$. If this fails, then let $x_{n} \rightarrow^{\left(\mathrm{ru}^{*}\right)} \theta$ and $x_{n} \nrightarrow^{\mathcal{T}} \theta$. Since the cone is $\mathcal{T}$-normal, there is a full neighborhood $U$ of $\theta$ and a subsequence $\left(y_{k}\right)$ of $\left(x_{n}\right)$ such that $y_{k} \notin U$ for each $k$. Since $x_{n} \rightarrow^{\left(\mathrm{ru}^{*}\right)} \theta$ there is a subsequence $\left(y_{k_{i}}\right)$ of $\left(y_{k}\right)$ for which $y_{k_{i}} \rightarrow^{(\mathrm{ru})} \boldsymbol{\theta}$. Hence there exists an element $z \in X$ and a sequence $\left(\lambda_{i}\right)$ of real numbers decreasing to 0 with $-\lambda_{i} z \leqslant y_{k_{i}} \leqslant \lambda_{i} z$ for each $i$. Since $\lambda_{i} z \rightarrow^{\mathcal{T}} \theta$, for sufficiently large $i_{0},-\lambda_{i_{0}} z$ and $\lambda_{i_{0}} z$ are elements of $U$, and thus $y_{k_{i_{0}}} \in U$ since $U$ is full, but this contradicts the choice of $\left(y_{k}\right)$. The proof of the last statement of the proposition follows the outline of the proof of the first statement in an obvious manner.

The proof of $\left(1^{\prime}\right) \Rightarrow(2)$ below is close to that of Peressini [12, Proposition 2.4].

LEMMA 2.2. Let $(X, \mathcal{T})$ be a complete, metrizable topological vector space ordered by $a \mathfrak{T}$-closed cone $K$. The following statements are equivalent:

(1) $K$ is generating.

(2) For each sequence $\left(x_{n}\right) \subset X$ and $x \in X, x_{n} \rightarrow^{\tilde{T}} x$ implies $x_{n} \rightarrow^{\left(\mathrm{ru}^{*}\right)} x$.

If, moreover, $X$ has property (A), then the following is also equivalent to each of (1) and (2):

(3) For each sequence $\left(x_{n}\right) \subset X$ and $x \in X, x_{n} \rightarrow^{\pi} x$ implies $x_{n} \rightarrow^{\left(0^{*}\right)} x$.

Proof. Condition ( 1 ) is equivalent to

( $\left.1^{\prime}\right)$ For each $\mathcal{T}$-neighborhood $U$ of $\theta, K \cap U-K \cap U$ is a $\mathcal{T}$-neighborhood of $\theta[5$, p. 106], [11, Theorem 5.3].

We first show $\left(1^{\prime}\right) \Rightarrow(2)$. Let $d$ be an invariant metric for $\mathcal{T}[7$, p. 48]. It will suffice to show that if $d\left(x_{n}, \theta\right) \rightarrow 0$ then $x_{n} \rightarrow^{\left(\mathrm{ru}^{*}\right)} \theta$. Let $\left(y_{k}\right)$ be a subsequence of $\left(x_{n}\right)$. Now $d\left(y_{k}, \theta\right) \rightarrow^{k} \theta$. Define $U_{i}=\left\{x \in X \mid d(x, \theta)<1 / i^{3}\right\}, i=1$, $2, \ldots$ Then for each $i, K \cap U_{i}-K \cap U_{i}$ is a neighborhood of $\theta$, so there is a subsequence $\left(z_{i}\right)$ of $\left(y_{k}\right)$ such that $z_{i} \in K \cap U_{i}-K \cap U_{i}, i=1,2, \ldots$ Write $z_{i}=u_{i}-v_{i}$ with $u_{i}, v_{i} \in K \cap U_{i}$ for each $i$. Hence $d\left(u_{i}, \theta\right)<1 / i^{3}$ and 
$d\left(v_{i}, \theta\right)<1 / i^{3}$ for each $i$. Using the invariance of $d$ and induction one has that $d\left(i u_{i}, \theta\right) \leqslant i d\left(u_{i}, \theta\right)<1 / i^{2}$, and similarly, $d\left(i v_{i}, \theta\right)<1 / i^{2}$ for each $i$. The sequence $\left\{\sum_{i=1}^{j} i u_{i}\right\}_{j=1}^{\infty}$ is Cauchy, since for $l \geqslant m$,

$$
d\left(\sum_{i=1}^{l} i u_{i}, \sum_{i=1}^{m} i u_{i}\right)<\sum_{i=m+1}^{l} \frac{1}{i^{2}} .
$$

Define $w_{1}=\sum_{i=1}^{\infty} i u_{i}$ and $w_{2}=\sum_{i=1}^{\infty} i v_{i}$. Since the cone $K$ is closed, $i u_{i} \leqslant w_{1}$ and $i v_{i} \leqslant w_{2}$ for each $i$. Hence $\theta \leqslant u_{i} \leqslant(1 / i) w_{1}$ and $\theta \leqslant v_{i} \leqslant(1 / i) w_{2}$ for each $i$. Therefore,

$$
-(1 / i)\left(w_{1}+w_{2}\right) \leqslant u_{i}-v_{i}=z_{i} \leqslant(1 / i)\left(w_{1}+w_{2}\right)
$$

for each $i$ so $z_{i} \rightarrow^{(\mathrm{ru})} \theta$ and hence $x_{n} \rightarrow^{\left(\mathrm{ru}^{*}\right)} \theta$.

$(2) \Rightarrow\left(1^{\prime}\right)$. We argue by contradiction. Suppose (2) holds and that there exists a neighborhood $U$ of $\theta$ such that $K \cap U-K \cap U$ is not a $\mathcal{T}$ neighborhood of $\theta$. Then there exists a sequence $\left(x_{n}\right) \subset X$ with $x_{n} \rightarrow^{\top} \theta$ and $x_{n} \notin K \cap U-K \cap U$ for each $n$. By (2) there is a subsequence $\left(y_{k}\right)$ of $\left(x_{n}\right)$ and an element $z \in K$ such that $-(1 / k) z \leqslant y_{k} \leqslant(1 / k) z$ for each $k$. Let $(1 / k) z-y_{k}=p_{k}$ for each $k$. Now $p_{k} \rightarrow^{\top} \theta$ and $(1 / k) z \rightarrow \theta$ and $y_{k}=(1 / k) z$ $-p_{k}$ with $(1 / k) z, p_{k} \in K$ for each $k$. Thus for sufficiently large $k_{0},\left(1 / k_{0}\right) z$ $\in U$ and $p_{k_{0}} \in U$, so $y_{k_{0}} \in K \cap U-K \cap U$, which is a contradiction.

Even without property (A) we have that (2) implies (3) since a closed cone provides an Archimedean order. Assuming that $X$ has property (A) the proof that $(3) \Rightarrow\left(1^{\prime}\right)$ parallels that of $(2) \Rightarrow\left(1^{\prime}\right)$ with $\left(\mathrm{ru}^{*}\right)$-convergence replaced by (o*)-convergence and $\left(\lambda_{k} z\right)$ replaced by a sequence $\left(z_{n}\right)$ with $\left(z_{n}\right) \downarrow \theta$.

Proof of TheOrem 1.2. The implication (1) $\Rightarrow(2)$ of Theorem 1.2 is given by Lemmas 2.1 and 2.2. We now show that $(2) \Rightarrow(1)$ in Theorem 2.1. Because of Lemma 2.2 it will suffice to show that the cone $K$ is $\mathcal{T}$-normal. Let $\left(x_{n}\right)$ and $\left(y_{n}\right)$ be sequences in $X$ such that $0 \leqslant x_{n} \leqslant y_{n}$ and $y_{n} \rightarrow^{\top} \theta$. The normality of $K$ will follow if it is shown that $x_{n} \rightarrow^{\top} \theta$. We show that $x_{n} \rightarrow^{\left(\mathrm{ru}^{*}\right)} \theta$. Let $\left(x_{n_{k}}\right)$ be a subsequence of $\left(x_{n}\right)$. Then $\left(y_{n_{k}}\right)$ is a subsequence of $\left(y_{n}\right)$ and, hence, $y_{n_{k}} \rightarrow \theta$. Applying (2) to $\left(y_{n_{k}}\right)$ there is a subsequence $\left(y_{n_{k_{1}}}\right)$ of $\left(y_{n_{k}}\right)$ for which $y_{n_{k_{1}}} \rightarrow^{(\mathrm{ru})} \theta$. Hence there is a sequence $\left(\lambda_{i}\right)$ of positive real numbers decreasing to 0 and an element $z \in K$ such that $-\lambda_{i} z \leqslant y_{n_{k_{1}}} \leqslant \lambda_{i} z$ for each $i$. Hence,

$$
-\lambda_{i} z \leqslant \theta \leqslant x_{n_{k_{i}}} \leqslant y_{n_{k_{i}}} \leqslant \lambda_{i} z
$$

for each $i$ so $x_{n_{k_{i}}} \rightarrow^{(\mathrm{ru})} \theta$. Hence $x_{n} \rightarrow^{\left(\mathrm{ru}^{*}\right)} \theta$, so by (2), $x_{n} \rightarrow^{\mathcal{T}} \theta$. Therefore $K$ is T-normal.

Proof of Theorem 1.4. The proof of $(1) \Rightarrow(2)$ is given by Lemmas 2.1 and 2.2. We now show (2) $\Rightarrow(1)$. We first show that the cone $K$ is $\mathcal{T}$-closed. So suppose that $\left(x_{n}\right) \subset K$ and $x_{n} \rightarrow^{\mathcal{T}} x \in X$. By (2), $x_{n} \rightarrow^{\left(\mathrm{o}^{*}\right)} x$, so $\left(x_{n}\right)$ has a subsequence $\left(x_{n_{k}}\right)$ such that $x_{n_{k}} \rightarrow^{(0)} x$. Hence there is a sequence $\left(y_{k}\right) \subset X$ with $y_{k} \downarrow x$ and $0 \leqslant x_{n_{k}} \leqslant y_{k}$ for each $k$. It follows that $x=\inf y_{k} \geqslant \theta$, so $x \in K$ and thus $K$ is $\Im$-closed. Next we observe that (2) implies that $X$ has 
property (A), for if $y_{n} \downarrow \theta$ then $y_{n} \rightarrow^{\left(\mathrm{o}^{*}\right)} \theta$, so by (2), $y_{n} \rightarrow^{\mathcal{T}} \theta$. That (2) implies $K$ is generating is given by Lemma 2.2. Finally, the proof that (2) implies $K$ is normal parallels that of the proof of Theorem 1.2 with $\left(\mathrm{ru}^{*}\right)$-convergence replaced by $\left(\mathrm{o}^{*}\right)$-convergence and the sequence $\left(\lambda_{i} z\right)$ by a sequence $\left(z_{i}\right) \subset K$ with $\left(z_{i}\right) \downarrow \theta$.

3. Applications to comparison of topologies. Following Luxemburg and Zaanen [9, p. 84] and Vulikh [13, p. 33] (using sequences instead of nets) we define three distinct order topologies.

Definition 3.1. Let $X$ be an ordered vector space. A subset $F$ of $X$ is (ru)-closed ((o)-closed) iff for each $\left(x_{n}\right) \subset F$ such that $x_{n} \rightarrow^{\text {(ru) }} x \in X\left(x_{n}\right.$ $\left.\rightarrow^{(0)} x \in X\right)$, we have $x \in F$. The (ru)-closed sets are the closed sets of a topology for $X$ called the relatively uniform topology which we denote by $\sigma_{\text {ru }}$. The (o)-closed sets are the closed sets of a topology, for $X$, the order topology, which we denote by $\mathscr{T}_{0}$. When the ordering is Archimedean it is clear that $\mathfrak{T}_{\mathrm{o}} \subset \mathfrak{T}_{\text {ru }}$. Let $\mathfrak{T}_{\mathrm{b}}$ denote the order bound topology for $X$. It is a locally convex topology which has as a local base the collection of all convex, balanced sets each of which absorbs each order bounded subset of $X$. It follows from Gordon [4, Corollary 3.5] that $\sigma_{b} \subset \mathcal{T}_{\text {ru }}$.

A proof of (2) of the following theorem is given in [6, p. 61] and also in [13, Theorem II.7.1, Theorem VI.3.2]. The only properties of o-convergence used in the proof are:

(i) If $x_{n} \rightarrow^{(0)} x$, then each subsequence $\left(x_{n_{k}}\right)$ satisfies $x_{n_{k}} \rightarrow^{(0)} x$.

(ii) If $x_{n}=x$ for each $n$, then $x_{n} \rightarrow^{(0)} x$.

(iii) If $x_{n} \rightarrow^{(\mathrm{o})} x$ and $x_{n} \rightarrow^{(\mathrm{o})} y$, then $x=y$.

Properties (i), (ii), and (iii) also hold for (ru)-convergence in Archimedean ordered vector spaces. Thus the same proof which yields (2) also gives (1) and we have the following theorem.

THEOREM 3.2. In an Archimedean ordered vector space $X$ the following equivalences hold:

For each $\left(x_{n}\right)$ and $x \in X$,

(1) $x_{n} \rightarrow^{\mathcal{T}_{\mathrm{ru}}} x$ if $x_{n} \rightarrow^{\left(\mathrm{ru}^{*}\right)} x$, and

(2) $x_{n} \rightarrow^{\mathcal{T}_{0}} x$ iff $x_{n} \rightarrow^{\left(\mathrm{o}^{*}\right)} x$.

THEOREM 3.3. Let $(X, \mathcal{T})$ be a topological vector space ordered by a $\mathcal{T}$-normal cone. Then $\mathcal{T} \subset \mathcal{T}_{\mathrm{ru}}$. If, in addition, $X$ has property $(\mathrm{A})$ then $\mathcal{T} \subset \mathcal{T}_{\mathrm{o}}$.

Proof. We show that if $F$ is $\mathcal{T}_{\text {-closed then } F}$ is $\mathcal{T}_{\mathrm{ru}}$-closed. Suppose $\left(x_{n}\right) \subset F$ and $x_{n} \rightarrow^{(\mathrm{ru})} x$. Then $x_{n} \rightarrow^{\left(\mathrm{ru}^{*}\right)} x$, so by Lemma $2.1, x_{n} \rightarrow^{\mathcal{T}} x$. Since $F$ is $\mathcal{T}_{-}$ closed, $x \in F$. Thus $F$ is $\sigma_{\text {ru }}$-closed. The proof that $\sigma_{\subset} \subset \sigma_{\mathrm{o}}$ is similar.

In the following theorem the conclusion that $\sigma_{b} \subset \mathcal{T}$ is due to Namioka [11, Theorem 5.4].

THEOREM 3.4. Let $(X, \mathcal{T})$ be a complete, metrizable topological vector space ordered by $a$ T-closed cone $K$. Then $K$ is generating iff $\mathcal{T}_{\mathrm{ru}} \subset \mathcal{T}$. Thus, since both 
$\mathcal{T}_{\mathrm{o}}$ and $\mathcal{T}_{\mathrm{b}}$ are subsets of $\mathcal{T}_{\mathrm{ru}}$, it follows that $\mathcal{T}_{\mathrm{o}}$ and $\mathcal{T}_{\mathrm{b}}$ are contained in $\mathcal{T}_{\text {when }} K$ is generating.

Proof. If $K$ is generating and $F$ is $\widetilde{T}_{\text {ru }}$-closed, and $\left(x_{n}\right) \subset F$, and $x_{n} \rightarrow^{\top} x$, then by Lemma $2.2, x_{n} \rightarrow^{\mathcal{T}_{\mathrm{ru}}} x$. Since $F$ is $\mathscr{T}_{\mathrm{ru}}$-closed, $x \in F$. Thus $F$ is $\mathcal{T}_{\text {-closed. }}$ Conversely, suppose $\mathcal{T}_{\text {ru }} \subset \mathcal{T}$. Then $x_{n} \rightarrow^{\sigma^{T}} x$ implies $x_{n} \rightarrow^{T_{\mathrm{ru}}} x$, so by Theorem $3.2, x_{n} \rightarrow^{\left(\mathrm{ru}^{*}\right)} x$, so by Lemma $2.2, K$ is generating.

The equivalence of (1) and (5) below is due to Namioka [11, Theorem 5.4].

TheOREM 3.5. Let $(X, \mathcal{T})$ be a complete, metrizable, topological vector space ordered by $a$ T-closed cone $K$. The following statements are equivalent:

(1) $K$ is generating and $\mathfrak{T}$-normal.

(2) $\sigma=\sigma_{\text {ru }}$.

(3) $\sigma \subset \sigma_{\text {ru }}$ and $K$ is generating.

(4) $\mathcal{T} \supset \mathcal{T}_{\mathrm{ru}}$ and $K$ is $\mathcal{T}_{\text {-normal. }}$

Moreover, if $(X, \mathcal{J})$ is locally convex, the following is also equivalent:

(5) $\mathscr{T}=\sigma_{\mathrm{b}}$.

Proof. (1) $\Rightarrow(2)$ is given by Theorems 3.3 and 3.4. Conversely, if (2) holds then $x_{n} \rightarrow^{\mathscr{T}} x$ iff $x_{n} \rightarrow^{\mathcal{T}^{\mathrm{ru}}} x$, so by Theorem 3.2, $x_{n} \rightarrow^{\mathcal{T}} x$ iff $x_{n} \rightarrow^{\left(\mathrm{ru}^{*}\right)} x$ and (1) follows by Theorem 1.2. (2) $\Leftrightarrow$ (3): If (2) holds then (1) $\Leftrightarrow$ (2) implies (3). Conversely, if (3) holds then Theorem 3.4 implies (2). (2) $\Leftrightarrow$ (4): If (2) holds then (1) $\Leftrightarrow(2)$ implies (4). Conversely, if (4) holds then Theorem 3.3 implies (2).

THEOREM 3.6. Let $(X, \mathcal{T})$ be a complete, metrizable topological vector space ordered by a cone $K$. The following statements are equivalent:

(1) $K$ is $\mathcal{T}$-closed, generating, and $\mathcal{T}$-normal, and $(X, \mathcal{T})$ has property (A).

(2) $\mathcal{T}=\sigma_{0}$.

(3) $\mathcal{T} \subset \mathcal{T}_{\mathrm{o}}$ and $K$ is $\mathcal{T}_{\text {-closed }}$ and generating.

(4) $\mathcal{T} \supset \mathcal{T}_{\mathrm{o}}, K$ is $\mathcal{T}$-normal, and $(X, \mathcal{T})$ has property (A).

Proof. (1) $\Leftrightarrow(2)$ : That (1) implies (2) is given by Theorems 3.3 and 3.4 . Conversely, if (2) holds it follows by Theorem 3.2 that $x_{n} \rightarrow^{\top} x$ iff $x_{n} \rightarrow^{\left(0^{*}\right)} x$ so (1) follows by Theorem 1.4. (2) $\Leftrightarrow$ (3): (2) $\Leftrightarrow$ (1) implies (3) and conversely (3) using Theorem 3.4 implies (2). (2) $\Leftrightarrow$ (4): (2) $\Leftrightarrow$ (1) implies (4) and conversely (4) using Theorem 3.3 implies (2).

COROllary 3.7. Let $(X, \mathcal{T})$ be a complete, metric topological vector space ordered by a $\mathcal{T}$-closed cone $K$. If $(X, \mathcal{T})$ has property (A) then the following are equivalent:

(1) $K$ is generating and $\Im-n o r m a l$.

(2) $\mathcal{T}=\sigma_{0}$.

(3) $K$ is generating and $\sigma \subset \mathcal{T}_{0}$.

(4) $K$ is $\mathcal{T}_{\text {-normal }}$ and $\mathcal{T}_{\mathrm{o}} \subset \mathcal{T}_{\text {. }}$

The above corollary was proved by Carter [2] with the additional hypothesis that $(X, \mathcal{T})$ is locally convex and $K$ is the cone of a Schauder basis. The 
equivalence of (1) and (2) of the corollary was proved earlier by Ceitlin [3] with the same hypothesis as Carter.

\section{REFERENCES}

1. G. Birkhoff, Lattice theory, 3rd ed., Amer. Math. Soc. Colloq. Publ., vol. 25, Amer. Math. Soc., Providence, R.I., 1967. MR 37 \#2638.

2. L. H. Carter, An order topology in ordered topological vector spaces, Trans. Amer. Math. Soc. 216 (1976), 131-144.

3. J. M. Ceïtlin, Unconditional bases and semiorderedness, Izv. Vysš. Učebn. Zaved. Mat. 1966, no. 2(51), 98-104; English transl., Amer. Math. Soc. Transl. (2) 90 (1970), 17-25. MR 33 \#6362; 41 \#191.

4. H. Gordon, Relative uniform convergence, Math. Ann. 153 (1964), 418-427. MR 28 \#3332.

5. G. J. O. Jameson, Ordered linear spaces, Lecture Notes in Math., vol. 141, Springer-Verlag, Berlin and New York, 1970.

6. L. V. Kantorovič, B. Z. Vulih and A. G. Pinsker, Partially ordered groups and partially ordered linear spaces, Uspehi Mat. Nauk 6 (1951), no. 3 (43), 31-98; English transl., Amer. Math. Soc. Transl. (2) 27 (1963), 51-124. MR 13, 361; 27 \#1517.

7. J. L. Kelley and I. Namioka, Linear topological spaces, Van Nostrand, Princeton, N.J., 1963. MR 29 \#3851.

8. V. L. Klee, Jr., Boundedness and continuity of linear functionals, Duke Math. J. 22 (1955), 263-269. MR 16, 1030.

9. W. A. J. Luxemburg and A. C. Zaanen, Riesz spaces, Vol. I, North-Holland, Amsterdam, 1971.

10. R. W. May, Comparison of order topologies with the topology of an ordered topological vector space, Doctoral Dissertation, Florida State Univ., Tallahassee, 1975.

11. I. Namioka, Partially ordered linear topological spaces, Mem. Amer. Math. Soc. No. 24 (1957). MR 20 \# 1193.

12. A. L. Peressini, Ordered topological vector spaces, Harper and Row, New York, 1967. MR 37 \#3315.

13. B. Z. Vulih, Introduction to the theory of partially ordered spaces, Fizmatgiz, Moscow, 1961; English transl., Noordhoff, Groningen, 1967. MR 24 \# A3494; 37 \#121.

Department of Mathematics, Walla Walla College, College Place, Washington 99324

Department of Mathematics, Florida State University, Tallahassee, Florida 32306 